\title{
FIGO Stage IIIC2
}

National Cancer Institute

\section{Source}

National Cancer Institute. FIGO Stage IIIC2. NCI Thesaurus. Code C96260.

A FIGO stage term that applies to gynecologic cancers. There is no FIGO stage IIIC2 for cervical cancer; for endometrial cancer, it refers to regional metastasis to para-aortic lymph nodes with or without metastasis to pelvic lymph nodes. 